\title{
Research on the mechanism of high power solid laser
}

\author{
Wenkai Huang, Yu Wu
}

Lab center, Guangzhou University, Guangzhou, Guangdong, China.

Keywords: solid state laser, high power, resonator.

\begin{abstract}
A laser resonator with a concave lens is designed to solve the thermal lens effect, in order to obtain the $1064 \mathrm{~nm}$ laser with high power and high beam quality. At first we analyzed the selection of the compensation lens. Then high power laser beams are obtained with a Nd:YAG laser resonator we designed. So an output power of $48 \mathrm{~W}$ at $1064 \mathrm{~nm}$ is obtained, when the concave focus is $250 \mathrm{~mm}$ and the output mirror transmittance is $20 \%$,.
\end{abstract}

\section{Introduction}

Nd:YAG laser pumped by a laser diode has drawn more and more attention in recent years, because of its advantages of high power, high efficiency, low cost, long service life, etc. It can be used in many fields, such as industrial production, scientific research, medical diagnosis and environmental monitoring. With the development of solid laser application, there are higher requirements for the output power, beam quality and stability. As one of the most important factors, the thermal lens effect gets more attention in design of resonant cavity [1-9].

In order to obtain laser beams with high power and high beam quality, we should compensate the thermal lens effect of laser rod. In this paper, we select an appropriate lens to compensate the thermal lens effect of the laser rod, and get an optimal design of Nd:YAG laser resonator. The beam waist curves (at the principal planes of the laser rod) is studied theoretically and experimentally. Experiment results show that, using an appropriate concave lens and optimal design of the resonator, a high power and high beam quality laser can be achieved. We obtain an output power of $48 \mathrm{~W}$.

\section{Optimal design of Nd:YAG laser}

The existence of the thermal lens in solid state lasers severely affects the beam quality, the output power and the stability of the resonator. One method to compensate the thermal lens effect is adding a concave lens in the cavity. We use the theory of the dynamically stable resonator[10-19] to optimize the resonator with a compensate lens. The dynamically stable resonator meets the following conditions: (1) The diameter of the TEM00 mode should be limited by the active material. (2) The resonator should be insensitive to pump-induced fluctuations of the rod's focal length. (3) The resonator modes should be fairly insensitive to mechanical misalignments. Two configurations are discussed here, a plano-plano cavity and a plano-plano cavity with a concave lens. Fig. 1 shows the schematic of Nd:YAG laser cavity with a concave lens.

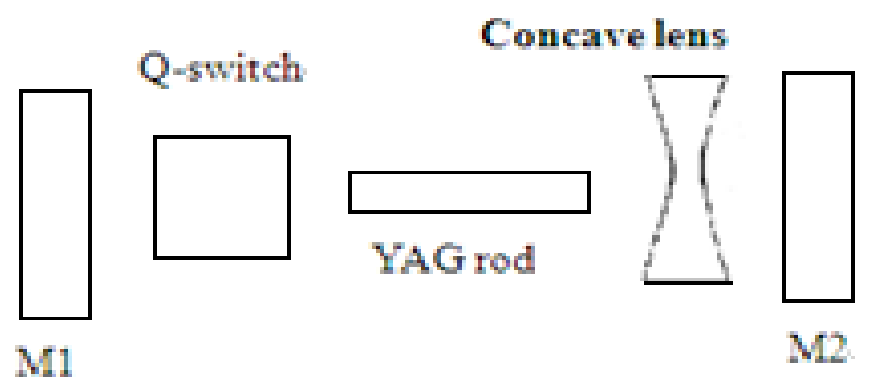

Fig.1. Schematic of Nd:YAG laser cavity

According to ABCD matrix theory, we calculate the beam radius wYAG at the principal plane of the laser rod (mode radius) with a concave lens (Fig. 2). Parameters used in calculation are: length 
of the total cavity is $286 \mathrm{~mm}$, length of the YAG laser rod is $120 \mathrm{~mm}$, the distance between YAG rod end faces and the concave lens is $10 \mathrm{~mm}$ and $90 \mathrm{~mm}$. The blank part of the figure is the unstable region. There are two stable regions in both cases. But only the above one is useful because the below one is narrow with a small thermal focus which is unrealistic. The low boundary of the above region in Fig. 2(a) is a line with thermal focus equal to $140 \mathrm{~mm}$. And that in Fig. 2(b) is a curve with thermal focus from $70 \mathrm{~mm}$ to $120 \mathrm{~mm}$ which is smaller than the previous case.

For achieving high power, high pump current is better, corresponding to a smaller thermal focus. Thus, a shorter distance between YAG rod end faces and the concave lens is better here. We choose the distance equal to $25 \mathrm{~mm}$ for mechanical constraint. High pump current from $17-24 \mathrm{~A}$ is expected to be used in the experiment for getting high power laser with corresponding thermal focus from $140 \mathrm{~mm}$ to $260 \mathrm{~mm}$. Thus, we choose a concave lens with $250 \mathrm{~mm}$ focus length according to Fig. 2.

With the above resonator parameters, the curve between wYAG and the refractive power of thermal lens D (inverse of the thermal focal length) are calculated and shown in Fig. 3. For comparing, the curve of removing the concave lens is shown in the same figure.

It shows that the laser has two stable regions. When adding concave lens into the cavity, the two stable regions move right due to the compensation of thermal lens effects. Then the laser could operate at higher pump current (larger

D) result in higher laser power. Moreover, the resonator with a concave lens

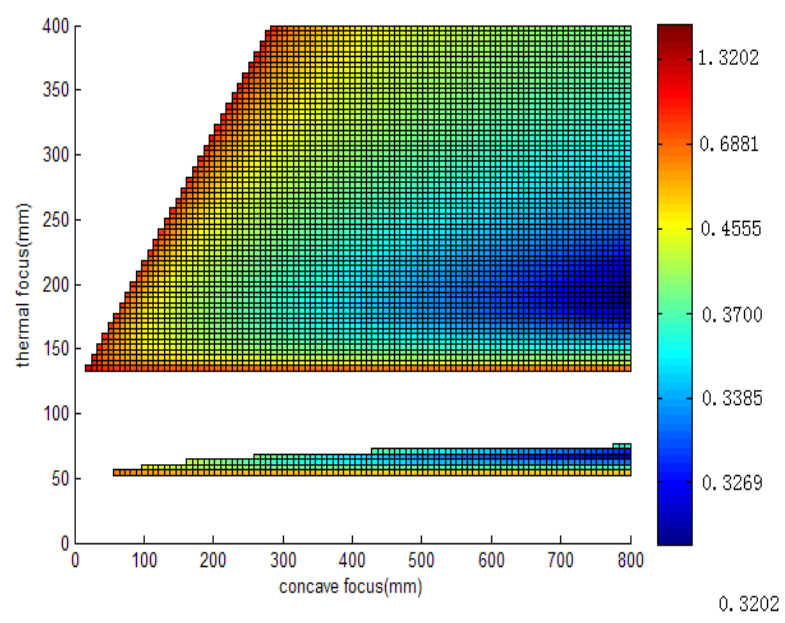

(a)

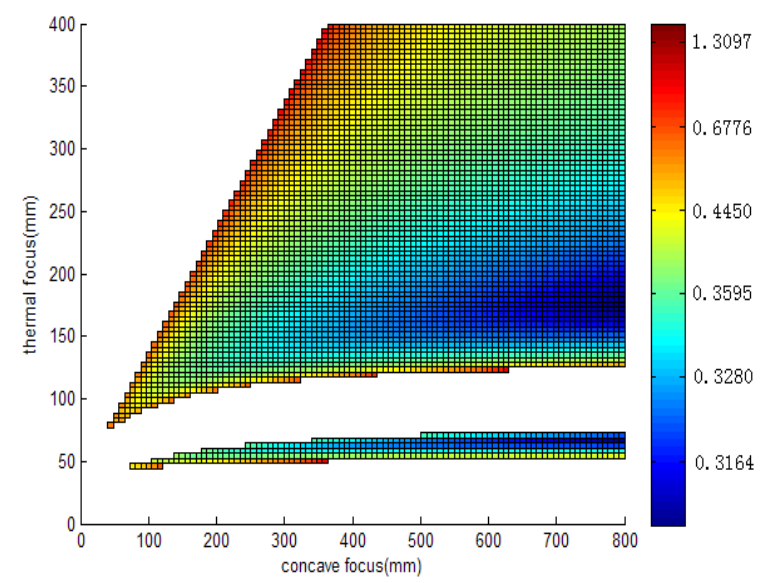

(b)

(a) the distance between the concave lens and the YAG rod end face is $90 \mathrm{~mm}$ (b)the distance between the concave lens and the YAG rod end face is $10 \mathrm{~mm}$ 
Fig.2. Stable region figure at different concave focus and thermal focus

has larger minimum beam radius $w_{\min }$ than the resonator without a concave lens. For achieving dynamic stability, lasers often operate at the state which $W_{\text {YAG }}$ is equal to $W_{\min }$.

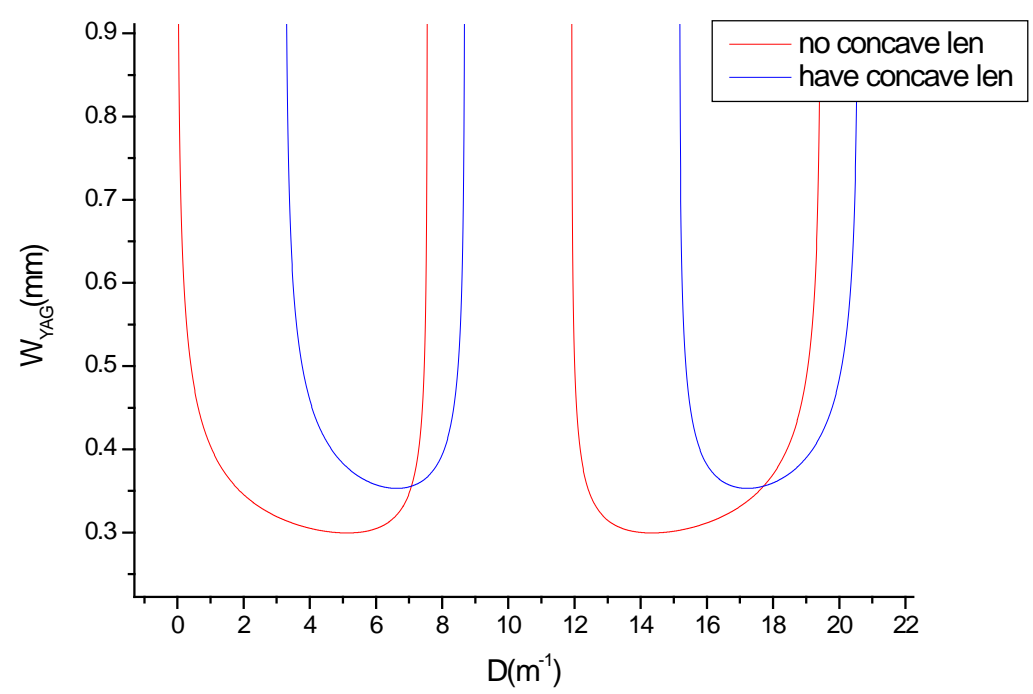

Fig.3. Refractive power versus beam waist radius at the main section of YAG laser rod

Figure 3 shows that the laser has two stable zones, the left corresponds to the second stable zone and the right corresponds to the first stable zone. When adding compensation lens into the cavity, both of the two stable zones move right. This makes the laser operating at high pump current to get high power laser. Because in the case of high pump current, the higher the LD pump power, the bigger the optical power of thermal lens. From figure 2 we can also see that after adding compensation lens the width of stable zone becomes narrow, the minimum beam waist radius increases accordingly.

Generally, the width of stable zone $\Delta D$ and minimum beam waist radius $W_{\min }$ satisfy the following relationship:

$$
w_{\min }^{2} \cdot \Delta D=\frac{2 \lambda}{\pi}
$$

The lasers have two stable areas and low pumping current is the first stable region while high pumping current is the second stable region. Experimental conditions limit the pump current can only be adjusted from $0 \mathrm{~A}$ to $24 \mathrm{~A}$, and therefore, the laser can only work in the second stable area. In calculation we found that, At the left edge (low current position) in the second stable region, misalignment sensitivity is very large, which indicates that the laser will be very unstable and can not work effectively when it just goes into stable region. However, with the increasing of pump current, misalignment sensitivity decreases rapidly. In the middle and right part of the second stable area, misalignment sensitivity has been close to the value of the first stable area, which illustrates that the laser is able to function properly. We use CCD to record the speckle pattern of laser leaked out by holophote M2 along with the changing of pump current, when the pump power is $21 \mathrm{~A}$, the horizontal and vertical cross-sectional shapes of the speckle are close to Gaussian distribution.

The resonator will be insensitive to pump-induced fluctuations of the rod's focal length ( $\mathrm{d} w_{\mathrm{YAG}}$ $/ \mathrm{dD}=0)$. According to the first conditions of dynamically stable resonator, larger $w_{\min }$ would get better beam quality. The beam quality factor can be estimated by [20] 


$$
M^{2}=\frac{d^{2}}{4 w_{t}^{2}}
$$

Where, $d$ is the diameter of laser rod, $w_{\mathrm{t}}$ is mode radius. Then submit the $w_{\min }$ and $d=4 \mathrm{~mm}$, we obtain $M^{2}=44.6$ without concave lens and $M^{2}=32.1$ with a concave lens. It is visible that after adding a concave lens, beam quality will significantly improve.

\section{Experimental research and analysis}

The experimental setup is shown in Fig.1. A commercial laser diodes side-pumped Nd:YAG module is used. It consists of a 4-mm-diameter, 120-mm-long Nd:YAG crystal. Both of its end faces are flat and antireflection coated at $1064 \mathrm{~nm}$. M2 is a flat mirror with highly reflection at $1064 \mathrm{~nm}$, and M1 is the output coupler mirror with an $80 \%$ reflectivity at $1064 \mathrm{~nm}$. The concave lens is $250 \mathrm{~mm}$ in focus length and both of its side faces are anti-reflection coated at $1064 \mathrm{~nm}$.

The performances of the laser with and without the concave lens are shown in Fig. 4. The slope efficiency of the laser with the concave lens is $60 \%$, which is much larger than that without the concave lens, which is $22 \%$. The output power increases linearly with the pump power. It obtains a maximum output of $48 \mathrm{~W}$ at a pump current of $20 \mathrm{~A}$. However, the efficiency of the laser without the concave lens is becoming saturated when the pump current reaches $17 \mathrm{~A}$.

\section{Conclusions}

In this paper, the method of using a concave lens to compensate the thermal lens effect is proposed. The theory of dynamically stable resonator is used in optimizing the laser cavity. Experiments are performed. The results show that a

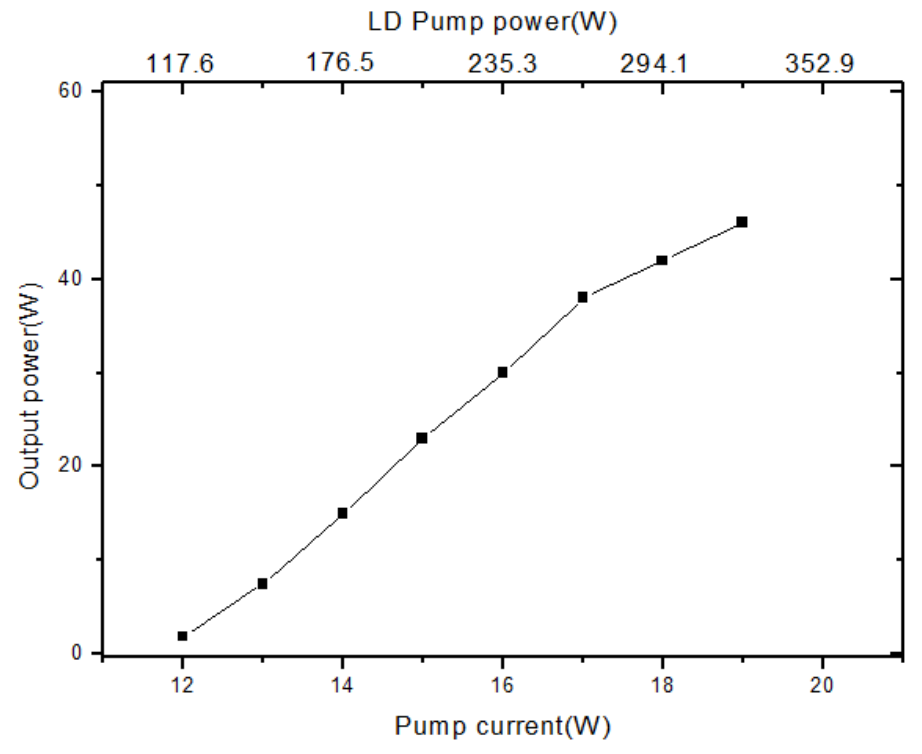

(a) 


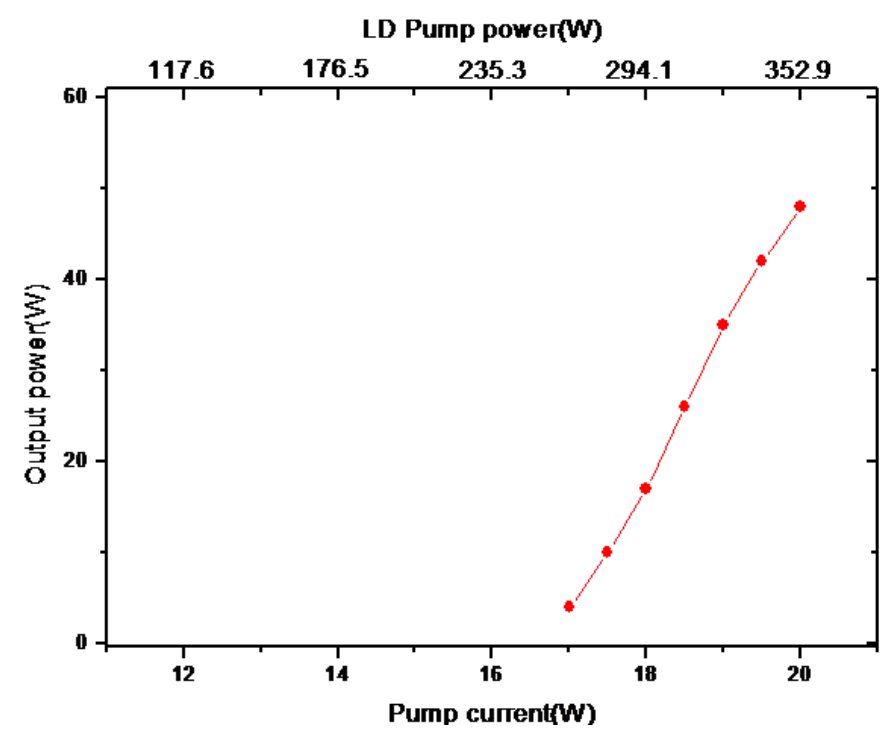

(b)

(a)the output power versus pump current without concave lens

(b)the output power versus pump current with a concave lens

Fig.4. The performances of the laser

laser with a concave lens has better performance than that without a concave lens. A maximum output of $48 \mathrm{~W}$ is obtained in our experiment.

\section{References}

[1] G.Y. He, J. Guo, B. Wang, Z.X. Jiao, Generation of radially polarized beams based on thermal analysis of a working cavity, Opt. Express 19 (2) , pp:18302-18309, 2011

[2] R. L. Niu, J. Liu, C. C. Liu, Y. Liu, L. J. Qin, Theoretical investigation on thermal lensing effects of Yb:KY(WO4)(2) in diode-pumped lasers, OPTIK, 122 (3) , pp:1931-1934,2011

[3] Y. Wang, K. Inoue, H. Kan, T. Ogawa, S. Wada, Study on thermally induced depolarization of a probe beam by considering the thermal lens effect, Source, J. Phys. D: Appl. Phys, 42 (5), pp:235108,2009

[4] H. Hao, Y. Zhang, Y. P. He, Thermal Effect of End-pumped Laser Crystal with Circular Cross-section, Chinese Journal of Lasers, 37 (6) , pp: 50-522010

[5] E. Wyss, M. Roth, T. Graf, Thermooptical Compensation Methods for High-Power Lasers, IEEE J. Quantum Electron, 38 (8) , pp:1620-16282002

[6] R. Zhou, Study on High-Power, High-Brightness All-Solid-State Green Laser and Ultraviolet Band Laser, Tianjin University, Master's thesis, 2004

[7] V. Magni, Resonators for solid-state lasers with large-volume fundamental modes and high alignment stability, Applied Optics, 25 (2) , pp: 107-1171986

[8] V. Magni, G.Valentini, S. D. Silvestri, Recent developments in laser resonator design, Optical and Quantum Electronics, 23 (1) , pp: 1105-11341991

[9] G. C. Lo, S. D. Silvestri, V. Magni, Output power limitations in CW single transverse mode Nd:YAG lasers with a rod of large cross-section, Optical and Quantum Electronics, 25 (2), pp:489-500 1993

[10] W.Koechner, Solid-state Laser Engineering, Beijing World Publishing Corporation, 2005

[11] S. M. Li, Laser Device Principle and Design, Beijing: National Defence Press, pp: 182-1831998

[12] Li Long, Gan An-sheng, Qi Bing et al. Thermal effect of LD end- pumped

Nd : YAG crystal with variable thermal conductivity. Laser Technology. 2012, 36(5):612 616.

[13] Zhong Ru- tao, Wang Yu- tao, Huang Zhi- jun, The developing status and application of high power laser, LASER JOURNAL,32(2),pp:4-7,2011 
[14] L. Quintino, A. Cost a, R. Miranda. Welding with high power $\mathrm{f}$ iber lasers- A preliminary study, Materials \& Design, 28(9), pp: 1231 - 1237,2006

[15] S Campbell,O Blomster,et al.Comparision of small fibre connectors for high-power transmission. Proceedings of SPIE the International Society for Optical Engineering . 2010

[16]Yuanyuan Fan, Bing He, Jun Zhou et al. Thermal effects in kilowatt all fiber MOPA. Opt. Express, 19(16),pp:15162-15172,2011

[17]D.C.Brown, H.J.Hoffman. Thermal, stress, and thermal optic effects in hgh average power double clad silica fiber lasers. IEEE J.Quantum Elcctron. 37(2), pp:207-217,2001

[18]B.Zintzen, T.Langer, J.Geiger et al. Heat transport in solid and air clad fibers for high power fiber lasers. Opt. Express, 15(25):16787-16792, 2007

[19]J.W.Kim, D.Y.Shen, K.Jayanta et al. Fiber laser pumped Er:YAG laser. IEEE J. Sel. Top. Quantum Electron. 15(2),pp:361-371,2009

[20] S. M. Zhang, Research on high-brightness all-solid-state laser and continuous-wave intracavity mid-infrared optical parametric oscillator based on PPLN, Tianjin University Master's thesis, pp:36-37,2007 